
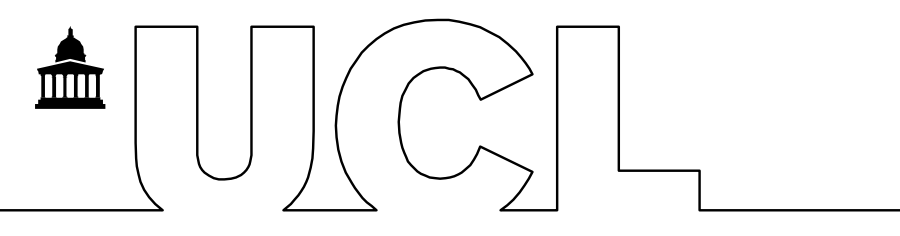

Coathup, MJ; Edwards, TC; Samizadeh, S; Lo, WJ; Blunn, GW; (2015) The effect of an alginate carrier on bone formation in a hydroxyapatite scaffold. Journal of Biomedical Materials Research Part B: Applied Biomaterials 10.1002/jbm.b.33395. (In press). Downloaded from UCL Discovery: http://discovery.ucl.ac.uk/1470091

\title{
ARTICLE
}

\section{The Effect of an Alginate Carrier on Bone Formation in a Hydroxyapatite Scaffold.}

Melanie J Coathup BSc, PhD', Thomas C Edwards MBBS, BSc ${ }^{1}$, Sorousheh Samizadeh BSc, PhD', Wei-Jen Lo BSc, PhD', Gordon W Blunn BSc, PhD'.

${ }^{1}$ John Scales Centre for Biomedical Engineering, Institute of Orthopaedics and Musculoskeletal Science, Division of Surgery and Interventional Science, University College London, The Royal National Orthopaedic Hospital, Brockley Hill, Stanmore Middlesex. HA7 4LP. United Kingdom.

${ }^{2}$ Wollaton Medical Consultancy Ltd, 3 Crowborough Ave, Nottingham, NG8 2RN. United Kingdom.

\section{Corresponding author and author for reprint requests.}

Coathup, MJ BSc, PhD ( ${ }^{*}$ Corresponding Author).

John Scales Centre for Biomedical Engineering, Institute of Orthopaedics and Musculoskeletal Science, Division of Surgery and Interventional Science, University College London, The Royal National Orthopaedic Hospital, Brockley Hill, Stanmore Middlesex. HA7 4LP. United Kingdom. Tel: +44 208954 5264, Fax: +44 208420 7392, e-mail: m.coathup@ucl.ac.uk.

\section{Abstract}

This study investigated the osteoconductive properties of a porous hydroxyapatite (HA) scaffold manufactured using a novel technique similar to the bread-making process, alone and in combination with an alginate polysaccharide fibre gel (HA/APFG putty) and autologous bone marrow aspirate (BMA). The hypothesis was that the HA/APFG putty would be as osteoconductive as granular HA and that the presence of BMA would further enhance bone formation in an ovine femoral condyle critical defect model.

Thirty-six defects were created and either (1) porous HA granules, (2) HA/APFG putty or (3) HA/APFG putty + BMA were implanted. Following retrieval at 6 and 12 weeks, image analysis techniques were used to quantify bone apposition rates, new bone area, bone-HA scaffold contact and implant resorption.

At 6 weeks post-surgery, significantly lower bone apposition rates were observed in the HA/APFG putty group when compared to the HA $(p=0.014)$ and HA/APFG putty + BMA $(p=0.014)$ groups. At 12 weeks, significantly increased amounts of new bone formation was measured within the HA scaffold $(33.56 \pm 3.53 \%)$ when compared to both the HA/APFG putty $(16.69 \pm 2.7 \% ; p=0.043)$ and defects containing HA/APFG putty + BMA (19.31 $\pm 3.8 \% ; p=0.043)$.

The use of an alginate polysaccharide fibre gel as a carrier for injectable CaP bone substitute materials delayed bone formation in this model compared to HA granules alone which enhanced bone formation especially within the interconnected smaller pores. Our results also showed that the addition of autologous bone marrow aspirate did not further enhance its osteoconductive properties. Further work is 
Does an Alginate Gel Carrier Effect Bone Formation?

required to optimize the degradation rate of this alginate polysaccharide fibre gel binging agent before use as a directly injectable material used for bone defect repair.

Keywords: Bone regeneration; calcium phosphate; bone marrow aspirate; bone substitute materials.

\section{Introduction}

Increases in reconstructive orthopaedic surgery resulting from advances in surgical practice and the ageing population have led to an increase in the use of bone substitute materials, especially in oncologic surgery, traumatology, revision prosthetic surgery and in spinal surgery (1). Over two million bone grafting procedures are performed every year, and synthetic bone substitute materials are often used due to their ease of handling, excellent biocompatibility, improved safety profiles, low cost, availability and their ability to be used in a variety of clinical situations. The purpose of a graft material is to augment bony regeneration within a defect where the new bone formed should ideally penetrate and replace the graft through sequential remodelling, enabling the repair site to maintain an optimal balance between form and function (2). Currently, the "gold standard" in terms of bony integration is obtained using autograft due to its biological performance in terms of osteoinductivity (7 - 9) and osteoconductivity $(9-11)$. However disadvantages including limited availability and explant site pain and morbidity have reduced its use $(5,6)$. Allografts and xenografts which are only osteoconductive, can overcome these limitations, but the risk of immune reactions and in some countries, limited availability of tissue banks, patient compliance and regulatory restrictions are at present, significant problems $(12,13)$. Synthetic bone graft substitutes based on calcium phosphate materials are valid alternatives to tissue transplants and have been utilised clinically for over three decades, with varying efficacy and success (14). A limitation with current $\mathrm{CaP}$ bone substitute materials is that they often exist in a dry granular form, limiting handling ability during surgery (3). In the last 15 years,_injectable and moldable forms of bone substitute material, such as pastes and putties, have been developed as they offer many advantages including increased handling ability and are able to completely fill contained defects of complex geometric shapes (4) In addition and with the development of minimally invasive surgical methods, the requirement to treat bony defects with directly injectable biomaterials is increasing (3). The combination of hydrogels and calcium phosphate particles is emerging as a well-established trend for bone substitutes. Hydrogels are macromolecular networks with high hydrophilicity and interconnections between the polymeric chains. This structure allows them to swell without dissolution and although good cohesion between the hydrogel and $\mathrm{CaP}$ particles has been shown at the site of implantation, a balance with the viscosity is required as less viscous carriers are required for easier injectability. However this low viscosity may have a negative effect on shelf stability causing settling and phase separation upon injection (13). The degradation rate of the hydrogel matrix and the composite stiffness of the construct are both important as these factors have been shown to significantly influence vascularisation and cell influx within the biomaterial $(13,15-17)$. A hydrogel that degrades too slowly, may delay cell colonization and impede new bone formation as the cells are unable to penetrate the CaP scaffold in an appropriate time scale. Bone marrow aspirate (BMA) is a source of pluripotent cells and its use in combination with $\mathrm{HA}$ and $\beta$-TCP bone substitute materials has been shown to have a significant effect on increasing bone regeneration within the scaffold $(26-28)$. Although the number of stromal cells present within BMA are relatively low compared to treatments where these cells have been expanded in vitro, the direct use of BMA in augmenting bone healing avoids regulatory issues and is significantly less expensive (36).

The aims of this study were two-fold. The first aim was to investigate whether the addition of an alginate polysaccharide fibre gel binding agent will have an effect on the osteoconductive properties of a porous HA scaffold manufactured using a novel technique similar to the bread-making process. The second aim was to investigate whether bone formation within a porous HA scaffold with binding agent, would be further augmented by the addition of autologous stromal and progenitor cells. The hypotheses of this study were that the HA/APFG putty biomaterial would be as osteoconductive as granular HA and that the 
Does an Alginate Gel Carrier Effect Bone Formation?

presence of BMA would further enhance the osteoconductive properties in an ovine femoral condyle critical defect model at 6 and 12 weeks.

\section{Materials and Methods \\ Surgery}

A total of thirty-six cylindrical, critical sized defects measuring $8 \times 15 \mathrm{~mm}$ were created in the medial condyle of both the left and right femur of nine skeletally mature commercially cross-bred adult female sheep weighing between 65 and $80 \mathrm{~kg}$ and aged between 2 and 5 years. Three experimental groups were investigated at two time-points (6 and 12 weeks) post-operatively; (1) porous HA granules, (2) porous HA granules within an alginate polysaccharide fibre gel binding agent (HA/APFG putty) and (3) porous HA granules within an alginate polysaccharide fibre gel combined with autologous bone marrow aspirate (HA/APFG putty + BMA). Positions were rotated such that no implant combination was placed in the same location in each sheep more than once. All implants were provided sterile and prepackaged by Orthogem Ltd (Nottingham, UK) and this study investigated the osteoconductive properties of TriPore ${ }^{\circledR}$ HA (Orthogem Ltd, Nottingham, UK). TriPore ${ }^{\circledR}$ is a phase pure hydroxyapatite (crystallinity > $85 \%$ ) with a multilayered interconnected porous architecture that mimics the natural composition of bone. TriPore ${ }^{\circledR}$ consists of a unique three pore structure consisting of interconnected macropores (100 - 800 $\mu \mathrm{m}$ diameter), midipores (10 - $100 \mu \mathrm{m}$ diameter) and microspaces (1 - $10 \mu \mathrm{m}$ diameter) (Figure 1). TriPore $\AA$ has a total macroporosity of $80 \%$ and granules used in this study were irregularly shaped and measured between $2-4 \mathrm{~mm}$ in size. The granule packing characteristics were not measured in this study. The porosity in these granules was developed by a novel process whereby yeast was added to a mixture of carbon hydrate powder, HA powder and water to form a dough-like material. The porous structure in the scaffold was generated in a process similar to bread-making using a warm oven to make a green structure. The porous materials were then fired up in a high temperature furnace to remove any carbon hydrate materials and organic matter in order to maintain the porous structure within the HA block.

At the start of surgery, bone marrow aspirate was collected from the iliac crest and $1 \mathrm{ml}$ of aspirate combined with each $1 \mathrm{~cm}^{3}$ of HA/APFG putty prior to implantation. The HA/APFG putty was prepared by mixing a ratio of $0.045 \mathrm{~g}$ alginate dry fibre with $1 \mathrm{~cm}^{3}$ of $\mathrm{HA}$ granules and $1 \mathrm{~cm}^{3}$ of water (Figure 2). The putty formed a gritty, viscous paste that was molded into the defects by slight pressure and remained in place without flow and migration from the defect. In the non-putty group and prior to implantation, HA granules were gently immersed and coagulated in $10 \mathrm{mls}$ of venous blood to limit granule migration out of the defect. Prior to insertion, each wound was irrigated with sterile saline and the appropriate test material gently pressed into place. The location of each defect was marked by drilling and inserting two $1 \mathrm{~mm}$ sized tantalum beads on either side within adjacent bone. A second defect site was then created and separated by at least $1 \mathrm{~cm}$ of bone before the wound was closed and the procedure repeated on the contralateral side. Two implants were inserted into each femoral condyle and a total of four inserted into each animal. This means that at least one implant was repeated twice in each animal. This was not necessarily always the control, however every animal had a control defect present. Repeats of 6 implants per group at each of the 6 and 12 week time-points were investigated. All procedures were carried out following Ethics approval granted by the Royal Veterinary College and in compliance with the United Kingdom Home Office regulations [Animal Scientific Procedures Act (1986)]. Following surgery, animals were allowed immediate and full mobilization as tolerated. Antibiotic and analgesic prophylaxis was administered daily with subcutaneous injections of Baytril (Enrofloxacin $5 \mathrm{mg} / \mathrm{kg}$; Bayer AG Leverkusen) and Finadyne (Flunixin Meglumine $2 \mathrm{mg} / 45 \mathrm{~kg}$; Schering-Plough) for 3 days post-surgery. Animals were kept in individual pens for 1 week post operatively before being group housed. Two fluorochrome bone markers Oxytetracyline (30 mg/kg) and Calcein Green (30 mg/kg) were administered at weeks 2 and 4 in the six week group, and 8 and 10 weeks in the twelve week group. Oxytetracylcine and Calcein Green localize at sites of mineralization and when viewed under ultra-violet light, fluoresce 
Does an Alginate Gel Carrier Effect Bone Formation?

an orange and green colour respectively. The mean measurable distance between cement lines provides an assessment of bone apposition rates ( $\mu \mathrm{m} /$ day) within defects in each of the groups.

On retrieval, condyles were placed in $4 \%$ paraformaldehyde solution before being processed for undecalcified histology. Following dehydration in serial dilutions of alcohol, specimens were defatted and embedded in hard grade acrylic resin (LR White, London Resin Company, Reading, UK). Radiographs were taken and the location of each defect identified by the position of adjacent tantalum beads. Thin sections $(\sim 70 \mu \mathrm{m})$ were prepared by making longitudinal cuts through the centre of each defect using a grinding and polishing technique (EXAKT, Norderstedt, Germany). Samples were stained with Toluidine Blue and Paragon, which stained the soft tissue and bone respectively. In each of the three experimental groups investigated, total new bone area, bone-HA scaffold contact and scaffold resorption were quantified. Six random regions of interest $(\mathrm{ROI})$ were image captured using a $5 x$-magnification lens where three ROIs were located at the periphery and three within the centre of each defect site (Axiovision Release 4.6, Carl Zeiss, Jena, Germany). The line intercept method was used and a mask of interconnecting lines measuring $10 \times 12 \mathrm{~mm}$ was superimposed on top of each image and the type of material (mineralised bone, soft tissue or scaffold) at the intersection of each line was determined (total of 225 intercept points per image). Assessments were made to evaluate the proportion of mineralised bone formed, soft tissue and implant material within each of the 6 regions in each defect. The percentage of bone ingrowth is a percentage of the total area of the substitute material and not the just the available area for ingrowth. For bone-HA scaffold contact, the type of tissue at the interface was determined at the points where lines intersected with the biomaterial surface. The size of the pores was measured using image analysis. Quantification of bone in pores $>10 \mu \mathrm{m}$ was measured using the lineintercept technique and expressed as a percentage, however bone within pores $<10 \mu \mathrm{m}$ was not measured as these sized pores occurred predominantly within the struts of the scaffolds. Data was quantified and compared between groups.

\section{Scanning Electron Microscopy}

Thin sections were sputter-coated with a layer of gold palladium and viewed using Backscattered electron microscopy (JOEL JSM-5500LV; Jeol, Welwyn Garden City, UK).

\section{Statistical Analysis}

Analysis of the data was performed using SPSS software (v10.1; SPSS, Chicago, Illinios). A Kolmogorov-Smirnov test showed the data obtained was non parametric and the Mann-Whitney $U$ test was used for statistical comparison between experimental groups. The Kruskal-Wallis test with post-hoc Mann Whitney $U$ was used to compare data at different time points within one experimental group. $p$ values $<0.05$ were considered significant.

\section{Results}

\section{Bone Apposition Rates}

At 6 weeks post surgery, the slowest rate of bone apposition was measured in the HA/APFG putty group where no bone turnover was observed in any of the specimens (Figure 3). Apposition rates where higher in the HA $(1.18 \pm 0.68 \mu \mathrm{m} /$ day $)$ and HA/APFG + BMA $(0.53 \pm 0.53 \mu \mathrm{m} /$ day $)$ groups however no significant differences between these three groups were found. In all groups and at 12 weeks post operatively, bony apposition rates had significantly increased when compared to the HA/APFG putty group at the 6 week time point; HA $(1.40 \pm 0.13 \mu \mathrm{m} /$ day; $p=0.011), \mathrm{HA} / \mathrm{APFG}+\mathrm{BMA}(1.53 \pm 0.19$ $\mu \mathrm{m} /$ day; $p=0.011)$ and HA/APFG putty $(1.28 \pm 0.11 \mu \mathrm{m} /$ day; $p=0.008)$. No other significant differences were found when rates were compared at the 6 and 12 week time points.

\section{New Bone Area}


Does an Alginate Gel Carrier Effect Bone Formation?

At 6 weeks post surgery, highest amounts of new bone formation was measured within the HA granule group (18.30 $\pm 5.06 \%$ ) with least new bone measured within HA/APFG + BMA scaffolds $(8.57 \pm 2.90 \%)$, however no significant differences between groups was found (Figure 4). By 12 weeks, the amount of new bone formed had significantly increased in the HA (33.32 $\pm 2.74 \% ; p=0.05)$ and HA/APFG putty + BMA (19.32 $\pm 2.30 \% ; p=0.027)$ groups when compared to the 6 week time-point. Significantly highest amounts of new bone was measured in the HA granule scaffolds when compared to both the HA/APFG putty $(18.05 \pm 2.91 \% ; p=0.018)$ and HA/APFG putty + BMA $(p=0.016)$ scaffolds.

\section{Bone-HA Scaffold Contact}

At 6 weeks post surgery, highest amounts of bone-HA contact was measured in the HA granule group $(59.34 \pm 10.89 \%)$. Lowest values were seen in the HA/APFG putty + BMA group $(25.99 \pm 7.46 \%)$

(Figure 5). However, no significant differences were found. At 12 weeks post operatively, bone-HA scaffold contact had increased in all three groups, however a statistically significant increase was only seen in the HA/APFG putty + BMA group $(p=0.027)$. Significantly highest amounts of bone-HA scaffold contact was measured in the HA granule group $(72.65 \pm 3.38 \%)$ when compared to both the HA/APFG putty $(52.66 \pm 4.30 \% ; p=0.018)$ and HA/APFG putty + BMA groups $(54.01 \pm 6.31 \% ; p=0.047)$.

\section{Implant Area}

No significant differences were found when implant resorption was compared in the three groups over the 6 and 12 week post operative period (Figure 6).

\section{Qualitative Analysis}

Analysis using light microscopy showed that new bone formation had occurred in all groups at the 6 week time-point post surgery (Figure 7). Qualitatively, there appeared to be little difference in the way that the bone and soft tissue reacted to the different compositions. In all specimens, bone was closely associated with the Tripore ${ }^{\circledR}$ granule surface and appeared to form in a similar manner through intramembranous ossification. Bone formation was associated initially with the deposition of collagen fibres, often organized perpendicular to the implant and bone forming surface. These collagen fibres were $5-10 \mu \mathrm{m}$ in diameter. In all groups, bone formation was associated predominantly with the concentric walls of the pores and then filled the pore in a centripetal manner.

At 12 weeks, and in most specimens in the HA/APFG putty and HA/APFG putty + BMA groups, residual carrier was evident within the intergranular spaces (Figure 8). Samples in both groups showed no signs of an inflammatory reaction at either of the time-points investigated. Overall, increased amounts of bone formation was seen adjacent to the Tripore $\AA^{\circledR}$ granule only group however in all groups, mature lamellar bone was observed bridging granules together. Light microscopy showed areas of vascularization in all groups at both time-points with blood vessels evident both adjacent to the HA granules and within the pore structure. No obvious differences in the amount of vascularization was seen when all groups were compared at the same time point. Backscattered scanning electron microscopy showed that at both the 6 and 12 week time points and in all groups, bone formation was seen in pores as small as $10 \mu \mathrm{m}$ in size (Figure 9). Occasionally and in localized regions and with all three implant types, debris associated with fragmentation of the HA scaffold was observed within macrophages and multinucleate cells found close to the surface of the implant.

\section{Discussion}

A disadvantage in the use of many of the current calcium phosphate based bone substitute materials is that they exist as a hard and brittle material that is difficult to mold into the desired defect shape. During surgery, this often requires the surgeon to reshape the surgical site around the shape and structure of the implant causing an increase in bone loss, trauma to the surrounding tissue and longer surgical time (18). In addition, filling bone voids with individual particles may lead to incomplete filling of the defect or 
Does an Alginate Gel Carrier Effect Bone Formation?

granule dispersion and loss during surgery. Using flowable or moldable pastes or putties in which the binder or gel is added to the calcium phosphate ceramic granules, offers several clinical and economical advantages including the use of minimally invasive techniques where directly injectable materials may allow complete filling of the defect site thereby avoiding voids which can lead to fibrous encapsulation (19). Adding a polymer binder to osteoconductive calcium phosphate ceramic would appear the method of choice, but this may be detrimental to its osteoconductive potential. The aim of this study was to investigate the use of an injectable calcium phosphate bone graft material combined within an alginate polysaccharide fibre gel carrier. A commercially available porous HA scaffold (Tripore® Nottingham, UK) was combined with an alginate polysaccharide fibre gel and bone formation within the scaffold compared to and without autologous bone marrow within critical sized defects in an ovine model. The critical sized bone defect is defined as the smallest sized osseous wound that will not spontaneously heal with bone tissue (20). In the ovine model, a defect size of $8 \times 15 \mathrm{~mm}$ is considered critical $(21,23)$ and these defects have been successfully used for the placement of materials in the evaluation of the bone healing response to bone substitutes $(21-23)$.

A hydrogel carrier may improve surgical handling, allow direct delivery into the defect site and maintain graft placement, however it must be biocompatible with both the surrounding tissue, provide a suitable environment for bone regeneration and must not interfere with the bone healing mechanism. Optimal carriers should resorb within the first few days of implantation otherwise its presence may interfere with the initial bone healing phase by impeding cell influx, the interaction of connective tissue, vascularisation within the HA intergranular space and fast bone growth $(3,13,33)$. Turner et al. (29) investigated the use of three formulations of injectable calcium sulphate-based putties containing deminseralised bone matrix and a hydroxypropylmethylcellulose binding agent. Results showed greater amounts of bone formation when compared to control defects and they concluded that the putties investigated were an effective delivery means for bone graft materials in bony defect repair. Another study by Turner et al. (30) investigated bone regeneration in a canine critical size defect model and showed that the same putty was as effective as autograft in healing bone at 6,13 and 26 weeks post surgery. A study by Urban et al. (31) also reported successful bone healing with calcium sulphate-based materials within a hydroxypropylmethyl cellulose carrier in a canine model at 13 and 26 weeks post operatively. In contrast, an in vitro study reported by Sohier et al. (32) investigated the use of a silated hydroxypropylmethyl cellulose hydrogel as a carrier for calcium phosphate ceramics and showed that following three dimensional culture, human bone marrow derived mesenchymal stem cells remained viable and retained their osteoblastic differentiation potential, however were not able to proliferate or colonise the constructs. They concluded that the hydrogel was not suitable for bone tissue engineering as the gel did not present a micro-environment with any recognisable binding sites allowing cells to attach, migrate and proliferate. The same group compared crosslinked and non-crosslinked formulations of the carrier in a critical size femoral epiphysis defect in rabbits, and concluded that the carrier delayed cell colonisation and new bone formation (33). They suggested that this was because the carrier degraded too slowly, impeding cell penetration into the construct within an appropriate time-scale, as shown in their in vitro study, even though the carrier degraded without release of cytotoxic products (34). A similar outcome was recently reported in an ectopic bone formation ovine model where five different hydrogels were investigated as carriers for calcium phosphate bone substitute materials. They reported a general trend where bone formation increased into the gaps between the CaP granules in carriers that dissolved faster (35). These studies suggest that an inert chemistry, rapid dissolution and clearance of the polymer binder surrounding the microarchitecture of the CaP granules is required to allow for optimal bone formation.

In our study, bone apposition rates, percentage new bone area and bone-HA scaffold contact increased in all groups at the 12-week time-point when compared to 6 weeks post operatively. A study by Hing et al. (24) investigated bone apposition rates and bone formation within porous HA granules (without a carrier) in a bony defect in rabbits at 3,12 and 24 weeks. Results showed an increased rate of bone 
Does an Alginate Gel Carrier Effect Bone Formation?

mineralisation with increasing time post-operatively. The authors suggested that the difference maybe due to blood vessel infiltration within the graft area and increased protein adsorption to the scaffold. Bone formation begins with the formation of an apatite layer which enhances protein adsorption resulting in the differentiation of osteoprogenitor cells into osteoblasts leading to the deposition of bone (25). In order for the apatite layer to form, the bone graft material must first come into contact with blood, which facilitates the dissolution of the graft and its re-precipitation onto the surface $(14,24,25)$. In addition, blood is an abundant source of proteins, which would further enhance this process. Qualitative results from our study showed fewer areas of blood vessel formation in the 6 week groups when compared to samples at 12 weeks post surgery and this may be the reason why a significantly reduced bone apposition rate, new bone area and bone-HA scaffold contact was measured early on. In addition, bone apposition rates, percentage new bone area and bone-HA scaffold contact were all significantly decreased in the HA/APFG putty and HA/APFG putty + BMA groups at both of the time points investigated, and it is likely that the gel carrier present within the defects impeded connective tissue infiltration, angiogenesis and new bone formation. Increased bone area was measured in the two putty groups as the post-operative time increased, and it is possible that gradual degradation of the gel allowed increasing exposure of the HA scaffold to connective tissue penetration, angiogenesis and new bone formation. However, bone formation was significantly lower than with the granules alone, and this is because the putty had not completely resorbed and had prevented bone formation within the pores.

Our study showed that tissue within the pores $<10$ micron in size was mineralised collagen. This is a remarkable observation because these pores are too small for osteoblasts. Yet, mineralised bone was present and we believe that this may have been associated with the flow of osteoid and the formation of collagen before mineralisation into the pores from nearby cells.

Bone marrow aspirate contains pluripotent mesenchymal stem cells and has been shown to have a significant effect on increasing bony healing within HA and $\beta$-TCP bone substitute materials to rates similar to autograft $(26-28)$. In our study, the incorporation of autologous BMA within the scaffold material was used to provide a source of stem cells and growth factors thereby potentially increasing the rate and amount of new bone formation. Results showed that the alginate polysaccharide fibre gel significantly inhibited new bone formation and bone-HA scaffold contact despite the presence of bone marrow within the defect.

In this study, we hypothesised that the HA/APFG putty would be as osteoconductive as granular HA and that the presence of BMA would further enhance bone formation in an ovine femoral condyle critical defect model. Results showed that the presence of the gel significantly impeded new bone formation and bone-HA scaffold contact and we can therefore reject our hypothesis. Limitations of this study were that the resorbablity of the APFG carrier was not quantified and the effect of BMA on augmenting bone growth within the porous HA granules without the gel was not investigated. Another criticism may be the low number of animals used, but we found statistical differences in both bone area and bone contact which were two of the three parameters measured in this study. A reasonable increase in the number of animals would not have shown any differences in bone apposition rates between the various groups as the $p$ value was high.

The delayed bone formation response seen in this study is important when considering the use of a putty for the injectable delivery of calcium phosphate granules in bone defect repair and a balance between handle ability during surgery, mechanical stability and cell influx needs to be further optimised. New putties and injectables used for the administration of bone substitute materials should be tested in vivo in an effective model and results from bone grafts incorporated within putties should be similar to bone formation in granules without the use of a carrier. 
Does an Alginate Gel Carrier Effect Bone Formation?

Conflicts of Interest: Orthogem Ltd supplied the graft materials and funded this study. Authors from the John Scales Centre were involved in the study design, data collection, analysis and interpretation. Dr Wei-Jen Lo has received payments or services either directly or indirectly (via Orthogem Ltd) in support of this work. No author has had any other relationships, or has engaged in any other activities that could be perceived to influence what is written in this work.

\section{References}

1. Bhatt RA, Rozental TD. Bone graft substitutes. Hand Clin 2012;28(4):457-68.

2. Vallet-Regi M, Arcos D. Silicon substituted hydroxyapatites: A method to upgrade calcium phosphate based implants implants. J Mater Chem 2005;15:1509-151.

3. Low KL, Tan SH, Sein SHS, Roether JA. Calcium phosphate-based composites as injectable bone substitute materials. J Biomed Mater Res Part B: Appl Biomater 2010;94B:273-286.

4. $\quad \mathrm{Kim} \mathrm{JH}$, Oh JH, Han I, Kim H-S, Chung SW. Grafting using injectable calcium sulphate in bone tumour surgery: comparison with dimineralised bone matrix-based grafting. Clinics in Orthop Surgery 2011;3:191-201.

5. Habibovic P, De Groot K. Osteoinductive biomaterials - properties and relevance in bone repair. J Tissue Eng Regen Med 2007;1:25-32.

6. Nagineni VV, James AR, Alimi M, Hofstetter C, Shin BJ, Njoku I, Tsiouris AP, Hartl R. Silicatesubstituted calcium phosphate ceramic bone graft replacement for spinal fusion procedures. Spine 37(20):E1264-1272, 2012.

7. Urist MR. Bone formation by autoinduction. Science 1965;150(698):893-899.

8. Brown KL, Cruess RL. Bone and cartilage transplantation in orthopaedic surgery. A review. J Bone Joint Surg Am 1982;64(2):270-279.

9. Damien CJ, Parsons JR. Bone graft and bone graft substitutes: a review of current technology and applications. J Appl Biomater 1991;2(3):187-208.

10. Cornell CN, Lane JM. Current understanding of osteoconduction in bone regeneration. Clin Orthop 1998;355:S267-273.

11. Lane JM, Tomin E, Bostrom MP. Biosynthetic bone grafting. Clin Orthop 1999;367:S107-117.

12. R.E. Marx. Bone and bone graft healing. Oral Maxillofac Surg Clin North Am, 2007;19(4):455466.

13. D'Este M, Eglin D. Hydrogels in calcium phosphate moldable and injectable bone substitutes: sticky excipients or advanced 3-D carriers? Acta Biomaterialia 2013;9(3):5421-5430.

14. Hing K. Bone repair in the twenty-first century: biology, chemistry or engineering? Phil Trans $R$ Soc Lond A 2004;362:2821-2850.

15. Hydrogel/calcium phosphate composites require specific properties for three-dimensional culture of human bone mesenchymal cells. Acta Biomater, 2010;6(8):2932-2939. 
Does an Alginate Gel Carrier Effect Bone Formation?

16. J. Patterson, R. Siew, S.W. Herring, A.S.P. Lin, R. Guldberg, P.S. Stayton. Hyaluronic acid hydrogels with controlled degradation properties for oriented bone regeneration. Biomaterials, 2010;31(26):6772-6781.

17. G. Daculsi, A.P. Uzel, P. Weiss, E. Goyenvalle, E. Aguado. Developments in injectable multiphasic biomaterials: the performance of microporous biphasic calcium phosphate granules and hydrogels. J Mater Sci Mater Med, 2010;21(3):855-861.

18. Low K, Tan S, Zein S, Roether J, Mourino V, Boccaccini A. Calcium phosphate-based composites as injectable bone substitute materials. J Biomed Mater Res B Appl Biomater 2010;94(1):273-86.

19. Cardoso D, van den Beucken J, Both L, Bender J, Jansen J, Leeuwenburg S. Gelation and biocompatibility of injectable alginate-calcium phosphate gels for bone regeneration. J Biomed Mater Res A 2014;102(3):808-17.

20. Schmitz JP, Hollinger JO. The critical size defect as an experimental model for carniomandibulofacial nonunions. Clin Orthop Relat Res 1986;205:299-308.

21. Patel N, Brooks A, Clarke MT, Lee MT, Rushton N, Gibson IR, Best SM, Bonfield W. In vivo assessment of hydroxyapatite and silicate-substituted hydroxyapatite granules using an ovine defect model. J Mater Sci Mate Med 2005;16:429-440.

22. Coathup MJ, Cai Q, Campion C, Buckland T, Blunn GW. The effect of particle size on the ososteointegration of injectable silicate-substituted calcium phosphate bone substitute materials. $\mathrm{J}$ Biomed Mater Res B Appl Biomater 2013;101(6):902-10.

23. Campion CR, Chander C, Buckland T, Hing KA. Increasing strut porosity in silicate-substituted calcium-phosphate bone graft substitutes enhances osteogensis. J Biomed Mater Res B Appl Biomater 2011;97(2):245-54.

24. Hing KA, Annaz B, Saeed S, Revell PA, Bucklad T. Microporosity enhances bioactivity of synthetic bone graft substitutes. J Mater Sci Mater Med 2005;16(5):467-475.

25. Habibovic $P$, Yuan $H$, van der Valk $P$, van Blitterswijk CA, de Kroot K. 3D microenvironment as an essential element for osteoinduction by biomaterials. Biomaterials 2005;26(17):3565-3575.

26. Bansal S, Chauhan V, Sharma S, Maheshwari R, Juyal A, Rahuvanshi S. Evaluation of hydroxyapatite and beta-tricalcium phosphate mixed with bone marrow aspirate as a bone graft substitute for posteriorlateral spinal fusion. Indian J Orthop 2009;43(3):234-239.

27. Moro-Barrero L, Acebal-Cortina G, Suarez-Suarez M, Perez-Redondo J, Murcia-Mazon A, LopezMuniz A. Radiographic analysis of fusion mass using fresh autologous bone marrow with ceramic composites as an alternative to autologous bone graft. J Spinal Dis \& Tec 2007;20(6):409-415.

28. Jager M, Jelinek EM, Wess KM, Scharfstadt A, Jacobson M, Kevy SV, Krauspe R. Bone marrow concentrate: a novel strategy for bone defect treatment. Curr Stem Cell Res Ther. 2009;4:34-43.

29. Turner TM, Urban RM, Hall DJ, Infanger S, Gitelis S, Petersen DW, Haggard WO. Osseour healing using injectable calcium sulfate-based putty for the delivery of dimineralised bone matrix and cancellous 
Does an Alginate Gel Carrier Effect Bone Formation?

bone chips. Orthopaedics 2003;26(5S):571-575.

30. Turner TM, Urban RM, Hall DJ, Cheema N, Lim TH. Restoration of large bone defects using a hardsetting, injectable putty containing deminseralised bone particles compared to cancellous autograft bone. Orthopaedics 2003;26(5S):561-5.

31. Urban RM, Turner TM, Hall DJ, Infanger SI, Cheema N, Lim TH, Richelsoph K. An injectable calcium sulfate-based bone graft putty using hydroxypropylmethylcellulose as the plasticizer.

Orthopaedics 2004;27(1S):155-9.

32. Sohier J, Corre P, Weiss P, Layrolle P. Hydrogel/calcium phosphate composites require specific properties for three-dimensional culture of human bone mesenchymal cells. Acta Biomater 2010;6(8):2932-9.

33. Daculsi G, Uzel AP, Weiss P, Goyenvalle E, Aguado E. Development in injectable multiphasic biomaterials. The performance of microporous biphasic calcium phosphate granules and hydrogels. J Mater Sci Mater Med 2010;21(3):855-61.

34. Fellah BH, Weiss P, Gauthier O, Rouillon T, Pilet P, Daculsi G, Layrolle P. Bone repair using a new injectable self-crosslinkable bone substitute. J Orthop Res 2006;24(4):628-35.

35. Barbieri D, Yuan $\mathrm{H}$, de Groot F, Walsh WR, de Bruijn JD. Influence of different polymeric gels on the ectopic bone forming ability of an osteoinductive biphasic calcium phosphate ceramic. Acta Biomater 2011;7(5):2007-2014.

36. Conolly J, Guse R, Lippiello L, Dehne R. Development of an osteogenic bone-marrow preparation. J Bone Joint Surg71A:684-691, 1989.

\section{Figure Legends}

\section{Figure 1}

Electronmicrographs showing $[\mathrm{A}]$ the interconnected macroporous structure, [B] midipores and [C] microspaces among the annealed ceramic particles.

\section{Figure 2}

A photograph showing $[A]$ the porous HA granules, $[B]$ the HA APFG putty and $[C]$ a critical sized defect made within the medial femoral condyle.

\section{Figure 3}

Bone Apposition Rates ( $\mu \mathrm{m} /$ day) in all experimental groups at 6 and 12 weeks post surgery.

\section{Figure 4}

Percentage New Bone Formation within each of the three scaffolds at the 6 and 12 week post-operative time-points.

\section{Figure 5}

Percentage Bone-HA Scaffold contact within each of the three scaffolds at the 6 and 12 week postoperative time-points. 
Does an Alginate Gel Carrier Effect Bone Formation?

\section{Figure 6}

Percentage implant resorption over time.

\section{Figure 7}

Micrographs showing bone formation within HA scaffolds $[A]+[C]$, the HA/APFG putty scaffold $[B]$ and in [D], the HA/APFG putty + BMA group at 6 weeks post surgery.

\section{Figure 8}

Micrographs showing bone formation within HA scaffolds $[A]+[C]$, the HA/APFG putty scaffold $[B]$ and in [D], the HA/APFG putty + BMA group at 12 weeks post surgery.

\section{Figure 9}

Electronmicrographs showing bone formation within HA scaffolds at 12 weeks post surgery. The arrows point to bone formation within very small pores $(<10 \mu \mathrm{m})$. 